\title{
First Report of the Association of Myriogenospora atramentosa with the Plant Genus Hemarthria
}

\author{
Ko-Hsuan Chen, ${ }^{1}$ Ann Blount, ${ }^{1}$ Brittany Justesen, ${ }^{2}$ Joseph H. Walter, ${ }^{3}$ Marcelo Wallau, ${ }^{4}$ and Hui-Ling Liao ${ }^{1, t}$ \\ ${ }^{1}$ North Florida Research and Education Center, University of Florida, Quincy, FL 32351 \\ ${ }^{2}$ Osceola County Extension, University of Florida, Kissimmee, FL 34744 \\ ${ }^{3}$ Brevard County Extension, University of Florida, Cocoa, FL 32926 \\ ${ }^{4}$ Agronomy Department, University of Florida, Gainesville, FL 32611
}

Accepted for publication 17 October 2019.

Keywords: mycology, warm-season grasses, tangletop, forage, limpograss, Myriogenospora

Myriogenospora atramentosa is an epibiont fungus that produces black, linear stromata on the leaves of warm-season grasses including the genera Paspalum and Andropogon (Glenn et al. 1998). Infection of a grass by this fungus can lead to a disease called "tangletop," in which multiple leaves are tangled together by the fungal biomass (Glenn et al. 1998). M. atramentosa infections can also cause various degrees of host plant sterility (Glenn et al. 1998). Because $M$. atramentosa is in the family Clavicipitaceae containing several ergot alkaloid-producing fungi, its mycotoxin production and the potential risk to cattle health are of concern (Glenn et al. 1998).

Originally from southern Africa, limpograss (Hemarthria altissima) is a warm-season perennial grass that plays a key role in the cattle industry of the southeastern United States (Wallau et al. 2015). Compared with other warm-season grasses, limpograss is better adapted to poorly drained soil, more resistant to cold, and has greater digestibility over maturity, making it a good option for stockpiled forage (Kalmbacher et al. 1998; Wallau et al. 2015). As a result of these desired traits, the planted area of limpograss has expanded in Florida in the past few decades, covering over an estimated 200,000 ha (Wallau et al. 2015). Because limpograss is increasing in popularity as an improved forage species, understanding any potential plantpathogen interaction and its effect on the forage quality is essential.

Limpograss plants with tangletop signs and symptoms were collected in October 2018 from Osceola County, Florida, U.S.A. The infected, living grasses were retained in the greenhouse of North Florida Research and Education Center at Quincy, Florida. A dried specimen taken from the limpograss cultivar Floralta was deposited in the University of Florida Herbarium (accession FLASF-63888). Polymerase chain reaction was conducted targeting the nuclear ribosomal internal transcribed spacer (ITS) and large subunit (LSU) D1 regions (primers ITS1F and LR3) following U'Ren et al. (2010). Sanger sequencing was conducted, and the

${ }^{\dagger}$ Corresponding author: H.-L. Liao; sunny.liao@ufl.edu

Funding: This work was funded by the Florida Cattleman Enhancement Fund.

The author(s) declare no conflict of interest.

(C) 2019 The American Phytopathological Society obtained sequences were submitted to National Center for Biotechnology Information (NCBI) GenBank (ITS, MK953716; LSU, MK953717). To compare the obtained sequences with sequences in the public database, Basic Local Alignment Search Tool (BLAST) was performed in the NCBI portal (accessed May 2019).

M. atramentosa was confirmed to be associated with limpograss cultivar Floralta (Fig. 1). A similar tangletop morphology was also reported by photographs in the field for the cultivars Gitbuck and Kenhy, but these records require further microscopic and molecular confirmation. The infected plants of Floralta showed tangletop symptoms at an earlier stage of development (Fig. 2A). When the leaf of Floralta expanded and unfolded, the black, linear stroma appeared along the midrib of the leaf (Fig. 2A). The investigated asci produced on Floralta were unitunicate, and fusiform (Fig. 2B). Ascospores were at first cylindrical and later on broke into fusoid part-spores (Fig. 2C). Overall, the morphological characteristics of the teleomorph reported herein match previous descriptions of M. atramentosa (Glenn et al. 1998). However, M. atramentosa on limpograss sometimes merges several leaves consecutively along the stem, causing fungal signs that look like black line braids (Figs. $1 \mathrm{C}$ and $2 \mathrm{~A}$ ).

The top BLAST hits of ITS and LSU belonged to sequences previously identified as $M$. atramentosa and showed little to no divergence from the existing $M$. atramentosa sequences (Table 1). Although a wide range of host species have been reported for $M$. atramentosa (Glenn et al. 1998), all molecular sequences publicly available have been generated from samples collected in the eastern United States (Table 1). Research with broader sampling schemes is required to understand the global geographic distribution and genetic diversity of $M$. atramentosa. The $M$. atramentosa-H. altissima association has not been reported from ranches located in north Florida, so efforts should be made to carefully examine pastures of limpograss in that area in the future to ascertain the northern limit of the disease's occurrence.

M. atramentosa was considered to be restricted to plants in the new world (Glenn et al. 1998) (Fig. 3). However, H. altissima has a worldwide distribution (Fig. 3) and is also used as forage in Asia, South America, and Oceania (Xue et al. 2017). M. atramentosa is reported to be associated with the plant genus Hemarthria for the first time, through morphological and molecular investigations. Because of the increasing human usage, animal consumption, and global distribution of $H$. altissima, understanding its codistribution 

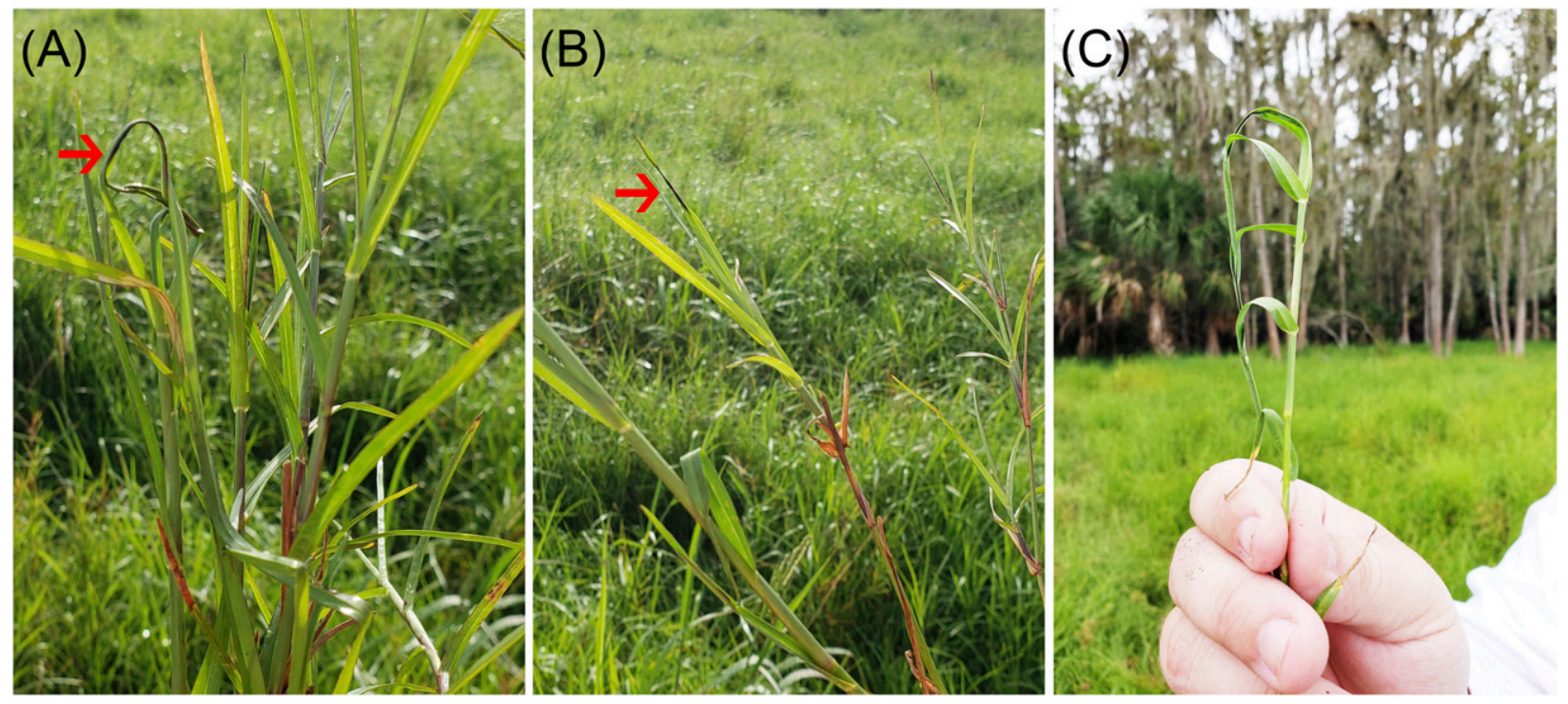

FIGURE 1

Limpograss (Hemarthria altissima) Floralta associated with Myriogenospora atramentosa in the field. A, Red arrow pointing to a tangletop condition caused by M. atramentosa stromata. B, Red arrow pointing to M. atramentosa stroma on the tip of a leaf. C, M. atramentosa causing a "black line braid" condition by merging multiple leaves along the stem.

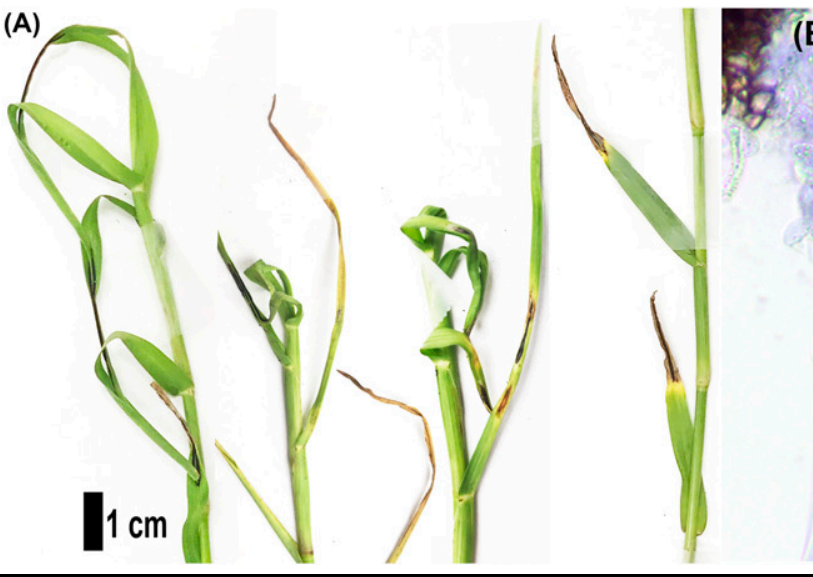

(B)

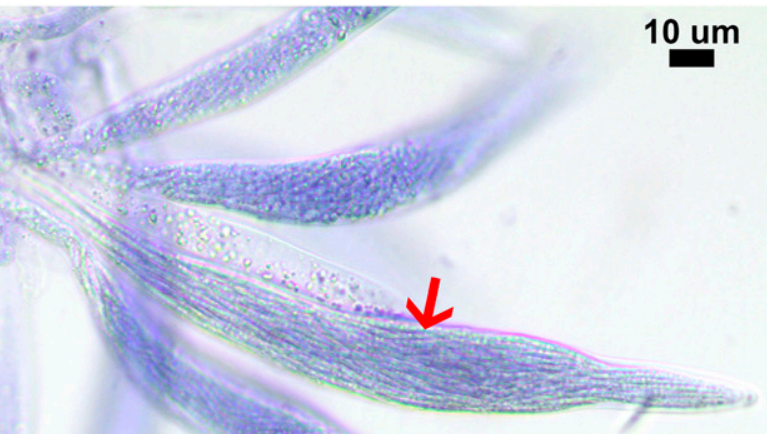

(C)

FIGURE 2

A, Limpograss (Hemarthria altissima) leaves infected by Myriogenospora atramentosa. B, Asci and ascospores of M. atramentosa stained with trypan blue. Red arrow pointing to fusiform asci containing many cylindrical ascospores. C, Fusoid part-spores.

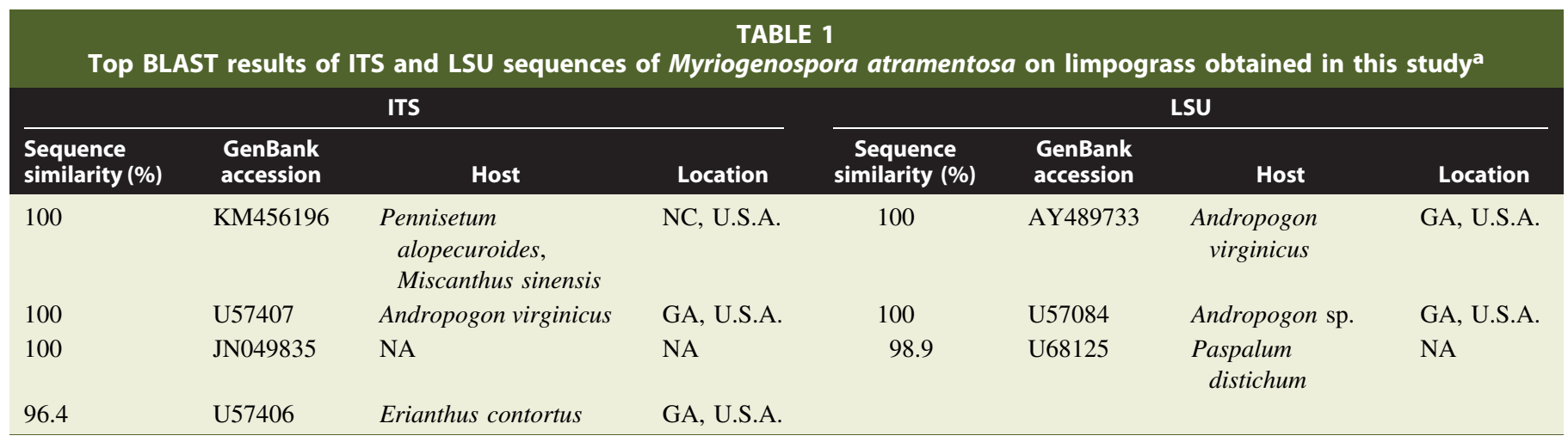

\footnotetext{
${ }^{a}$ Host information was derived from NCBI references. All BLAST hits shown were identified as $M$. atramentosa by the submitters. NA = not available.
} 


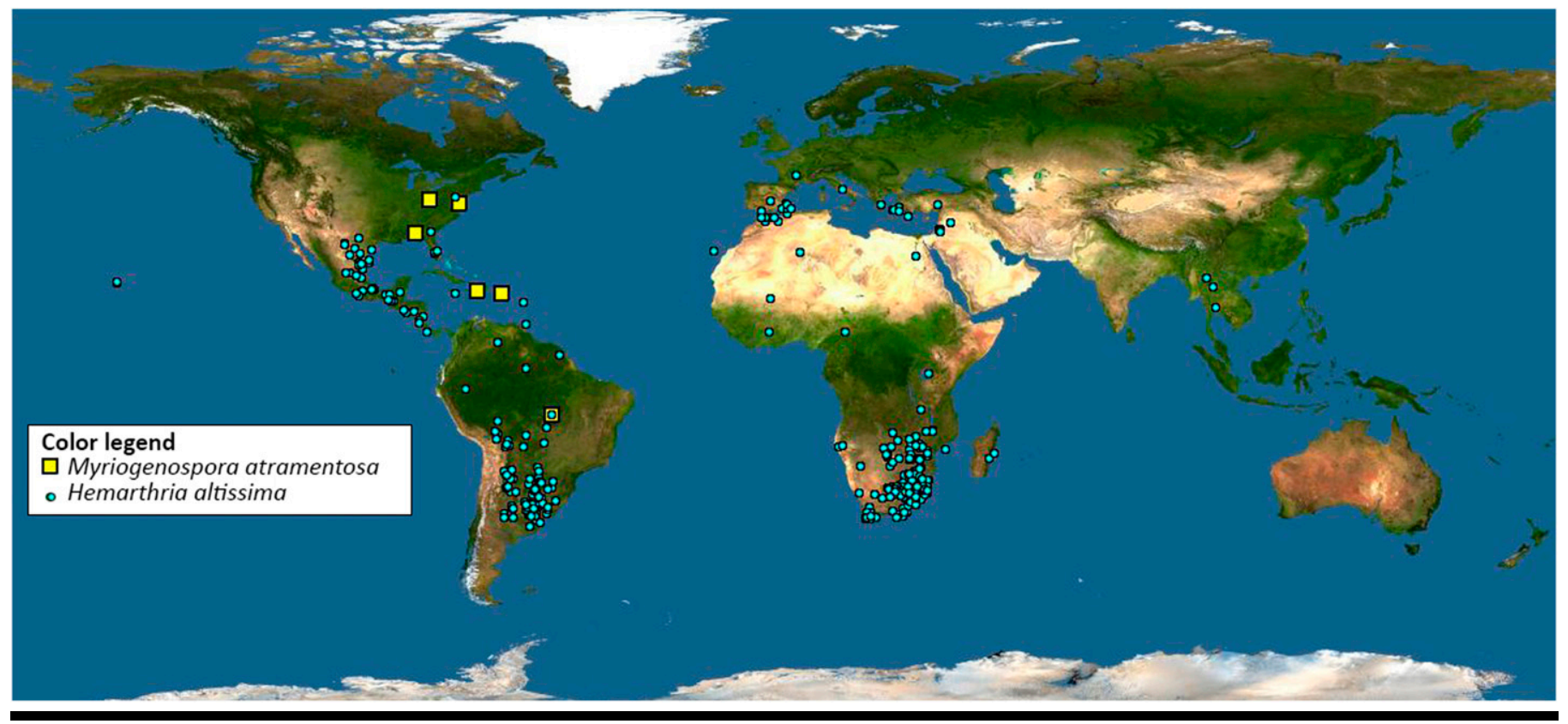

FIGURE 3

World distribution of Myriogenospora atramentosa and Hemarthria altissima. (Distribution map was produced by https://www.discoverlife.org/, accessed June 2019.)

with $M$. atramentosa and the potential mycotoxin content is critical to protect plants and animals.

\section{Literature Cited}

Glenn, A. E., Rykard, D. M., Bacon, C. W., and Hanlin, R. T. 1998. Molecular characterization of Myriogenospora atramentosa and its occurrence on some new hosts. Mycol. Res. 102:483-490.

Kalmbacher, R., Mullahey, J., and Hill, K. 1998. Limpograss and Hymenachne grown on flatwoods range pond margins. J. Range Manage. 51:282-287.
U'Ren, J. M., Lutzoni, F., Miadlikowska, J., and Arnold, A. E. 2010. Community analysis reveals close affinities between endophytic and endolichenic fungi in mosses and lichens. Microb. Ecol. 60:340-353.

Wallau, M. O., Sollenberger, L. E., Vendramini, J. M. B., Mullenix, M. K., Quesenberry, K. H., Gomide, C. A. M., Costa e Silva, V., and DiLorenzo, N. 2015. Herbage accumulation and nutritive value of limpograss breeding lines under stockpiling management. Crop Sci. 55:2377-2383.

Xue, L. H., Liu, Y., Wu, W. X., Liang, J., Zhang, L., Huang, X. Q., Zhou, X. Q., Johnson, R. D., Li, C. J., Wang, J. J., and Hu, J. Q. 2017. First report of leaf spot of Hemarthria altissima caused by Bipolaris zeae in China. Plant Dis. 101:243. 